Fixed Point Theory, 18(2017), No. 1, 223-228

http://www.math.ubbcluj.ro/ ${ }_{\text {nodeacj/sfptcj.html }}$

\title{
NOTE ON THE FIXED POINT PROPERTY
}

\author{
OSKAR GORNIEWICZ \\ Faculty of Mathematics and Information Science \\ Warsaw University of Technology \\ Koszykowa 75, 00-662 Warsaw, Poland \\ E-mail: oskgor@gmail.com
}

\begin{abstract}
In this paper it is proved that absolute approximative retracts and absolute multiretracts spaces have the fixed point property both for singlevalued continuous mappings and multivalued upper semi continuous mappings with $R_{\delta}$-values.

Key Words and Phrases: fixed point property, approximation theorems, set-valued mappings, absolute retracts, absolute approximative retracts.

2010 Mathematics Subject Classification: 47H04, 47H10, 54C60, 54H25.
\end{abstract}

\section{REFERENCES}

[1] K. Borsuk, Theory of Retracts, PWN, Warsaw 1967.

[2] L. Gorniewicz, Topological Fixed Point Theory of Multivalued Mappings, Springer, 2006.

[3] A. Granas, J. Dugundji, Fixed Point Theory, Springer, 2003.

[4] H. Ben-El-Mechaiekh, Spaces and maps approximation and fixed points, J. Comp. Appl. Math., 113(2000), 283-308.

[5] H. Noguchi, A generalization of absolute neighbourhood retracts, Kodai Math. Sem. Rep., 1(1953), 20-22.

[6] M.J. Powers, Fixed point theorems for non-compact approximative ANR-s, Fundamenta Math., 75, 61-68.

[7] R. Skiba, M. Slosarski, On a generalization of absolute neighbourhood retracts, Topology Appl., 156(2009), 697-709.

Received: April 14, 2015; Accepted: August 11, 2015.

The project was financed by funds of National Science Centre granted by decision number DEC2013/11/B/HS4/00857, carried out at University of Warsaw. 